\title{
GnRH Antagonist Protocol: Is It Optimal for All Patients of Different Ages Undergoing In Vitro Fertilization and Embryo Transfer?
}

\author{
Weijie Xing1, Haiyan Lin², Qingxue Zhang2* \\ ${ }^{1}$ Center for Reproductive Medicine, The Third Affiliated Hospital of Sun Yat-sen University, Guangzhou, China \\ ${ }^{2}$ Center for Reproductive Medicine, Sun Yat-sen Memorial Hospital of Sun Yat-sen University, Guangzhou, China \\ Email: *zhangqingxue666@aliyun.com
}

How to cite this paper: Xing, W.J., Lin, H.Y. and Zhang, Q.X. (2017) GnRH Antagonist Protocol: Is It Optimal for All Patients of Different Ages Undergoing In Vitro Fertilization and Embryo Transfer? Advances in Sexual Medicine, 7, 153-160. https://doi.org/10.4236/asm.2017.74012

Received: August 23, 2017

Accepted: September 18, 2017

Published: September 22, 2017

Copyright (c) 2017 by authors and Scientific Research Publishing Inc. This work is licensed under the Creative Commons Attribution International License (CC BY 4.0). http://creativecommons.org/licenses/by/4.0/

\section{Open Access}

\begin{abstract}
Background: To assess the effect of these two protocols in patients of different ages. Methods: 1923 in vitro fertilization and embryo transfer (IVF-ET) cycles were divided into two groups: a GnRH-ant protocol group and GnRH-a long protocol group, and then every group were subdivided into four age ranges. The general materials and IVF outcomes were compared. Results: The incidence of OHSS fluctuated from $0 \%$ to $2.37 \%$ with GnRH-ant protocol, which was significantly lower than another $(P<0.05)$. Of all age ranges, the implantation and clinical pregnancy rates with $\mathrm{GnRH}$-ant protocol were all lower than those with GnRH-a protocol. When the age was less than 35 years old, the CPRs in GnRH-ant protocol group were significantly lower than in another group (45.56\% vs. $62.29 \%$, $46.07 \%$ vs. $55.59 \%$, respectively; $P<0.05$ ). Conclusion: The antagonist protocol should be considered in patients with a high ovarian response (e.g., PCOS patients) to avoid OHSS. Older patients ( $>35$ years) could be treated with the antagonist protocol.
\end{abstract}

\section{Keywords}

GnRH Agonist, GnRH Antagonist, IVF-ET

\section{Background}

Controlled ovarian hyper-stimulation (COH) is an important step for IVF-ET, which can influence clinical outcomes and complications. In 1984, Porter and Craft used synthetic GnRH agonist in IVF-ET for the first time to inhibit the early luteinizing hormone (LH) surge and obtain a better clinical pregnancy rate (CPR) in IVF-ET [1]. Until now, the GnRH-a long protocol has been widely used. However, the inhibition of endogenous gonadotropin $(\mathrm{Gn})$ secretion re- 
quires large doses of exogenous Gn and prolonged Gn administration, which might result in OHSS and affect the fertility outcomes.

In the last decade, the GnRH antagonist (GnRH-ant) protocol has been used increasingly for IVF-ET [2]. In earlier times, its CPR rate was reportedly lower. Griesinger et al. reported that from IVF centers registered in Germany, the GnRH-ant protocol was only used in patients with recurrent IVF failure or of older age. Check et al. compared the GnRH agonist protocol with the antagonist protocol and found the CPR and delivery rates were similar [3].

Is the GnRH-ant protocol optimal for all IVF patients of different ages? It is still uncertain now. This research aimed to assess the effect of these two protocols in patients of different ages.

\section{Method}

1923 IVF/ICSI cycles from 2013-2015 were analyzed retrospectively. There were two groups in this study: a GnRH-ant protocol group (Group A) and a GnRH-a protocol group (Group B), and then each group was subdivided into four age ranges: $<31$ years, 31 to 35 years, 36 to 40 years, and $>40$ years. This retrospective study was approved by the Third Affiliated Hospital of Sun Yat-Sen University Reproductive Medicine Ethic Committee. The patient records/information was anonymized and de-identified prior to analysis.

GnRH-ant protocol was similar to the previous study [4]. On day 2 - 3 of menstrual cycle, the patients were received 37.5 IU-225 IU FSH (Gonal-F, Serono, Switzerland). When the diameter of dominant follicle met $14 \mathrm{~mm}$, dosage of $0.25 \mathrm{mg}$ GnRH-ant agonist (Cetrotide, Serono, Switzerland) was used per day. Triggering follicle maturation by human chorionic gonadotropin (hCG, $250 \mathrm{ug}$, Serono) or dual trigger (hCG 2000IU + GnRH-a $0.1 \mathrm{mg}$ ) was performed when two or more follicles reached a mean diameter of $18 \mathrm{~mm}$. 34 - 36 hours later, the patients were received oocyte retrieval.

In GnRH-a group, the patients received $1.0 \mathrm{mg}-1.3 \mathrm{mg}$ Triptorelin $(3.75 \mathrm{mg}$, Ferring) starting on day 20 of the previous cycle. FSH was used with dosage of 37.5 IU - 300 IU per day. After 34 - 36 hours with hCG trigger, ovum pick-up was done.

IVF or ICSI was performed. After 3 to 5 days, selected embryos were transferred. All patients received luteal phase support with $90 \mathrm{mg}$ progesterone vaginal gel (Crinone, Serono, UK) per day on the day of oocyte retrieval. Pregnancy was diagnosed by serum $\beta$-hCG 14 days later. Ultrasound with gestational sac could confirm clinical pregnancy.

\section{Statistical Analysis}

Data were analyzed by SPSS software. $t$-test was performed for means. $\chi^{2}$-test was for categorical variables. $P<0.05$ was defined as statistically significant.

\section{Results}

Patients' general data are showed in Table 1. The occurrences of OHSS fluctuated 
within $0 \%$ to $2.37 \%$ in Group A, which was significantly lower than Group B $(P<0.05)$.

Figure 1(a) and Figure 1(b) demonstrate that total doses of Gn and stimulation duration were significantly fewer in Group A than in Group B for each age range $(P<0.05)$. From Figure $1(\mathrm{a})$, we also found more and more gonadotropins were needed as age increased in Group B $(P<0.05)$.

Figure 2(a) shows that in the age range of $36-40$ years, the $E_{2}$ level was $2673.76 \pm 1396.72 \mathrm{pg} / \mathrm{mL}$ in Group A, which was significantly lower than in

Table 1. Demographic characteristics of patients in two groups.

\begin{tabular}{|c|c|c|c|}
\hline \multirow[b]{2}{*}{ Variable } & \multicolumn{2}{|c|}{ Groups } & \multirow[b]{2}{*}{$P$} \\
\hline & $\begin{array}{l}\text { GnRHant Group } \\
\text { (Group A) }\end{array}$ & $\begin{array}{l}\text { GnRHa Group } \\
\text { (Group B) }\end{array}$ & \\
\hline \multicolumn{4}{|c|}{ No. of total cycles } \\
\hline$<31$ years & 265 & 399 & I \\
\hline $31-35$ years & 295 & 368 & I \\
\hline 36 - 40 years & 185 & 109 & l \\
\hline$>40$ years & 129 & 173 & I \\
\hline \multicolumn{4}{|c|}{$\begin{array}{l}\text { No. of cancelled cycles because } \\
\text { of high risk of OHSS (n, \%) }\end{array}$} \\
\hline$<31$ years & $61.18 \%(52 / 85)$ & $76.47 \%(78 / 102)$ & $<0.05$ \\
\hline $31-35$ years & $33.65 \%(35 / 104)$ & $51.56 \%(33 / 64)$ & $<0.05$ \\
\hline 36 - 40 years & $9.72 \%(7 / 72)$ & $33.33 \%(4 / 12)$ & $<0.05$ \\
\hline$>40$ years & $2 \%(1 / 50)$ & $2.94 \%(1 / 34)$ & ns \\
\hline \multicolumn{4}{|c|}{ No. of OHSS (moderate and severe; $n, \%$ ) } \\
\hline$<31$ years & $2.26 \%(6 / 265)$ & $7.02 \%(28 / 399)$ & $<0.05$ \\
\hline $31-35$ years & $2.37 \%(7 / 295)$ & $5.98 \%(22 / 368)$ & $<0.05$ \\
\hline 36 - 40 years & 0 & $1.83 \%(2 / 109)$ & $<0.05$ \\
\hline$>40$ years & 0 & 0 & I \\
\hline \multicolumn{4}{|c|}{ No. of IVF cycles (n, \%) } \\
\hline$<31$ years & $67.17 \%(178 / 265)$ & $61.90 \%(247 / 399)$ & ns \\
\hline $31-35$ years & $69.15 \%(204 / 295)$ & $63.86 \%(235 / 368)$ & ns \\
\hline 36 - 40 years & $62.70 \%(116 / 185)$ & $60.55 \%(66 / 109)$ & ns \\
\hline$>40$ years & $65.89 \%(85 / 129)$ & $52.60 \%(91 / 173)$ & ns \\
\hline \multicolumn{4}{|c|}{ No. of embryos transferred } \\
\hline$<31$ years & $1.94 \pm 0.39$ & $2.00 \pm 0.37$ & $\mathrm{~ns}$ \\
\hline $31-35$ years & $1.99 \pm 0.38$ & $2.04 \pm 0.39$ & ns \\
\hline 36 - 40 years & $2.24 \pm 0.62$ & $2.68 \pm 0.51$ & $<0.05$ \\
\hline$>40$ years & $1.81 \pm 0.39$ & $2.59 \pm 0.62$ & $<0.05$ \\
\hline
\end{tabular}

Note: NS $=$ not statistically significant. Values presented as mean \pm SD unless otherwise specified. 


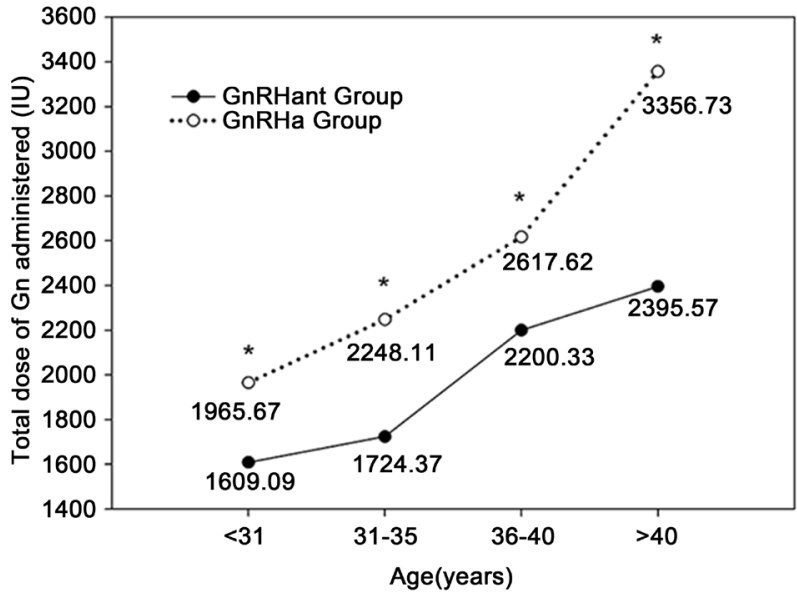

(a)

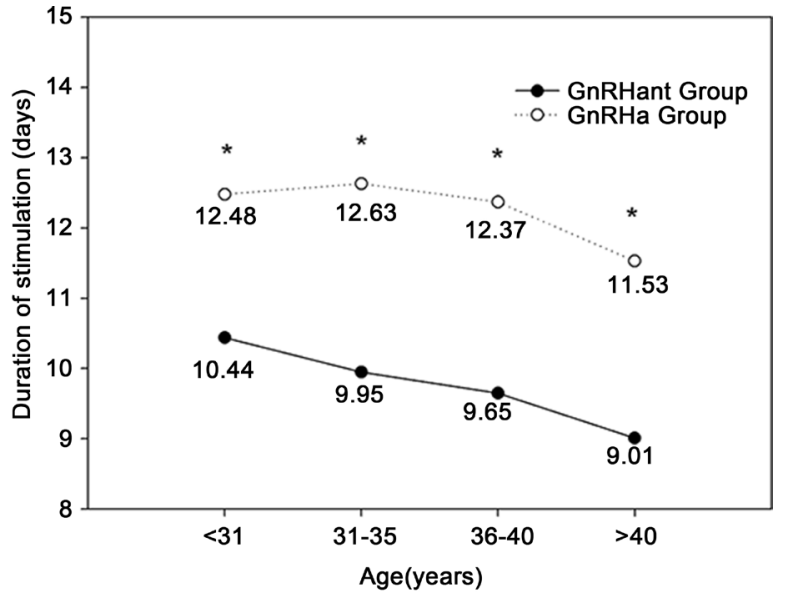

(b)

The doses of gonadotropin administration were significantly lower in GnRHant group than in GnRHa group in all the age ranges $(P<0.05)$. In all age ranges, the stimulation duration of the GnRHant protocol was significantly shorter than of the GnRHa long protocol $(P<0.05) .{ }^{\star} P<0.05$ versus the GnRHa group.

Figure 1. Comparison of total dose of Gonadotropins (a) and Stimulation duration (b).

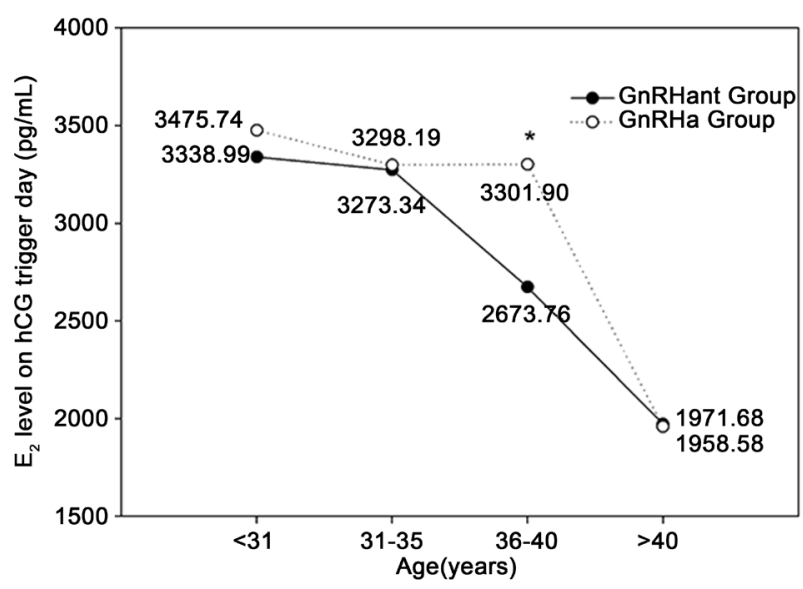

(a)

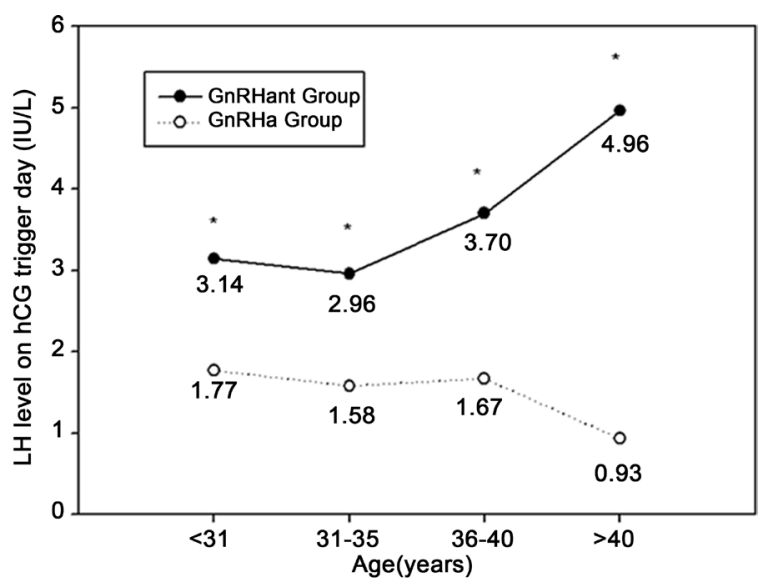

(b)

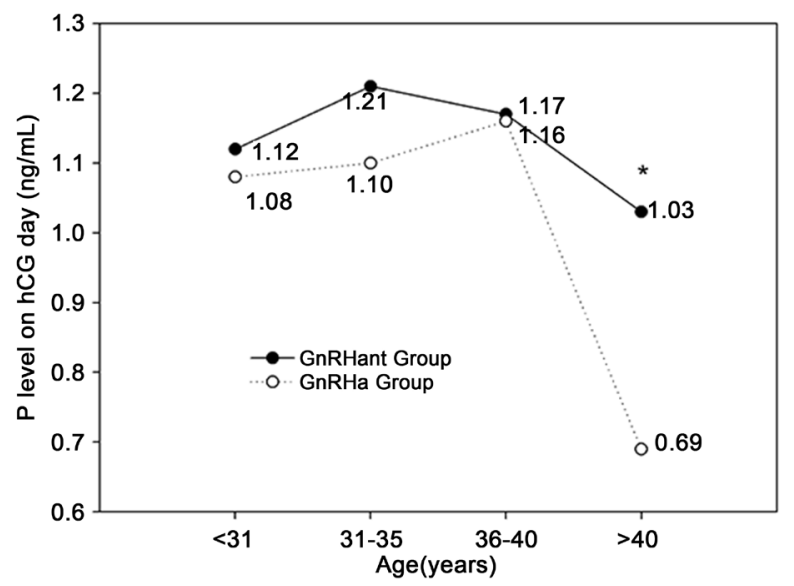

(c)

The differences of $\mathrm{E}_{2}$ levels in two groups were not significant in three age ranges (less than 31, 31 - 35 and over 40). In all age ranges, the LH levels were all significantly higher with GnRHant protocol than with GnRHa long protocol $(P<0.05)$. When patients' age was over 40 , the P levels in GnRHant group were significantly higher than in the GnRHa group $(P<0.05) .{ }^{*} P<0.05$ versus the GnRHa group.

Figure 2. Comparison of $\mathrm{E}_{2}$ levels (a), LH levels (b), and P levels (c) on the hCG trigger day. 


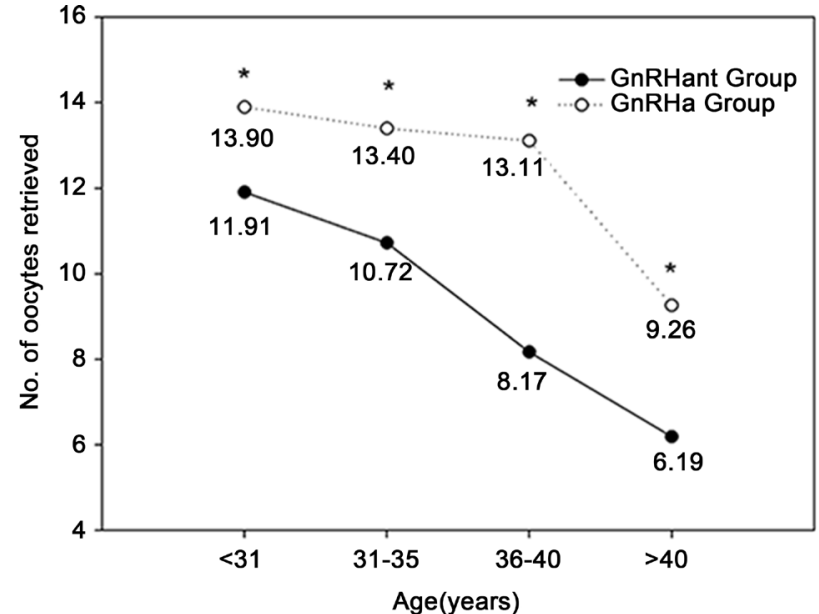

(a)

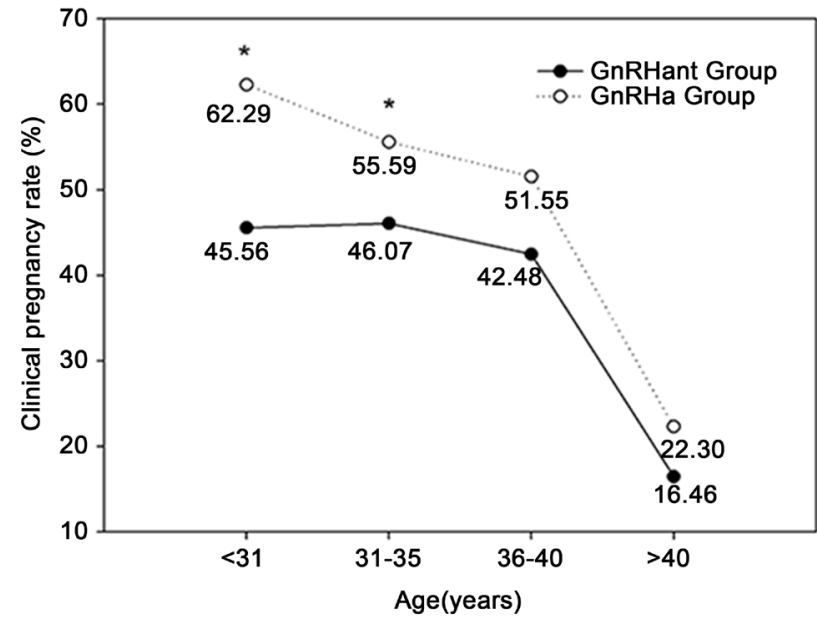

(c)

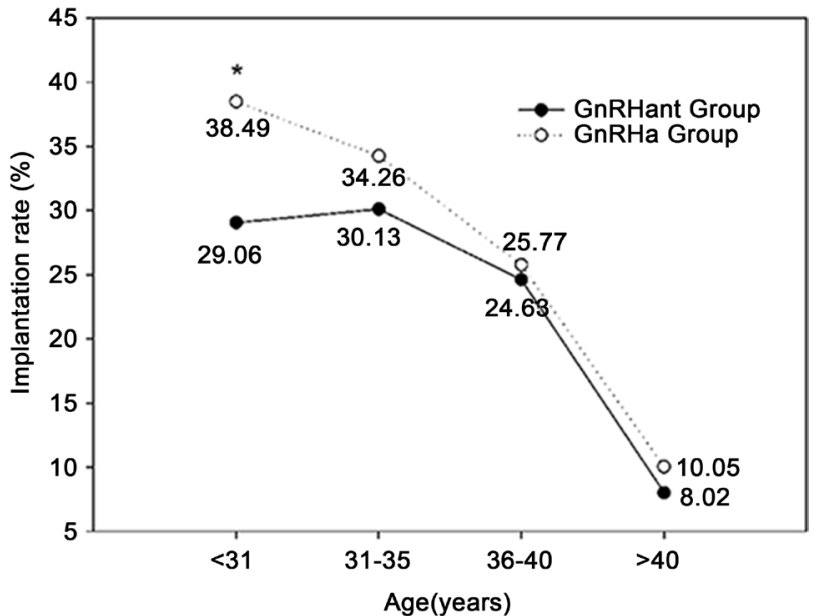

(b)

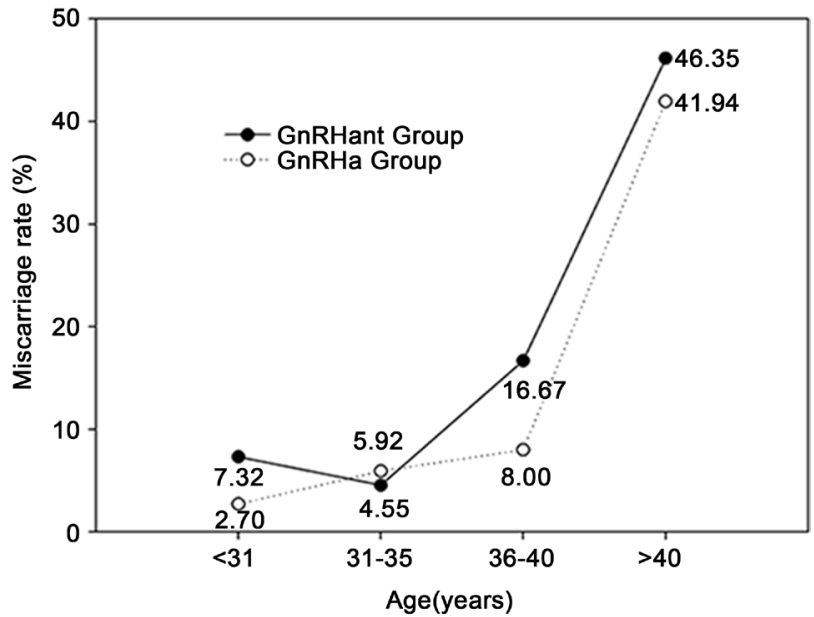

(d)

For every age range, the numbers of oocytes with GnRHant protocol were all significantly less than with GnRHa protocol $(P<0.05)$. The implantation rates with the GnRHant protocol were all lower than another protocol. The clinical pregnancy rates with the GnRHant protocol were all lower than another protocol for all age ranges. When age was less than 35 years old, the differences were significant $(P<0.05)$. The miscarriage rates of the two groups in every age range were all similar $(P>0.05) .{ }^{*} P<0.05$ versus the GnRHa group.

Figure 3. Comparison of retrieved oocytes (a), implantation rate (b), clinical pregnancy rate (c), and miscarriage rates (d).

Group B $(3301.90 \pm 1320.60 \mathrm{pg} / \mathrm{mL}, P<0.05)$. From Figure 2(b), we found that in Group A, the LH levels were all significantly higher than in Group B of four age ranges $(P<0.05)$.

As shown in Figure 2(c), in Group A, when patients' age was over 40, the $\mathrm{P}$ level on hCG trigger day was $1.03 \pm 0.49 \mathrm{ng} / \mathrm{mL}$, which was significantly higher than in Group B $(0.69 \pm 0.43 \mathrm{ng} / \mathrm{mL}, P<0.05)$.

Figure 3(a) shows that in every age range, the numbers of oocytes in Group A were all significantly less than those in Group B $(P<0.05)$. The numbers of oocytes significantly decreased with age in Group A $(P<0.05)$.

Figure $3(\mathrm{~b})$ and Figure $3(\mathrm{c})$ show that in all the age ranges, the implantation rates (IR) and clinical pregnancy rates (CPR) in Group A were all lower than those in Group B. However, the IR in Group A was significantly lower than in Group B, only when the age was less than 31 years old $(29.06 \%$ vs. $38.49 \%, P<$ 
0.05). When the age was less than 35 years old, the CPRs in Group A were significantly lower than in Group B $(P<0.05)$. The results analyzed by $\chi^{2}$-test list as follows: 1) As age increased, the CPR and IR in Group B decreased; 2) When patients' age was over 36 years, the IR of the two groups were both significantly decreased $(P<0.05) ; 3)$ In patients aged over 40 years, the CPRs in the two groups were significantly decreased $(P<0.05)$.

As shown in Figure 3(d), the miscarriage rates of Group A and B in each age group were similar $(P>0.05)$. By the $\chi^{2}$-test, we found that: 1$)$ In Group A, when a patient's age was greater than 36 years, the miscarriage rate significantly increased $(P<0.05) .2)$ When a patient's age was greater than 40 years in Group B, the miscarriage rate significantly increased $(P<0.05)$.

\section{Discussion}

$\mathrm{GnRH}$-ant protocol is a novel therapy for clinical ovarian stimulation, characterized by instant onset, reversible actions, and short administration [5] [6]. As shown in this study, the occurrence of OHSS in Group A was significantly lower than in Group B $(P<0.05)$. The major reasons for these outcomes could be: 1$)$ Without GnRH-a down-regulation in the antagonist protocol resulted in inferior follicular synchronization. Therefore, the $\mathrm{E}_{2}$ levels were much lower, leading to a lower risk of OHSS. Our results support the conclusions above. 2) GnRH-a could be substituted for hCG to stimulate the final maturation of the oocyte. $\mathrm{GnRH}-\mathrm{a}$ can directly induce luteolysis, leading to a rapid decrease in serum $\mathrm{E}_{2}$ levels and further lowering the incidence of OHSS [7] [8] [9]. It is likely to be the leading protocol for ovarian stimulation in the future [10] [11].

Several studies suggested that the antagonist protocol could reduce CPR to some extent [12]. Our data also yielded similar results. The reasons for the lower CPR with the antagonist protocol were: 1) The LH surge was more likely to occur. Our results indicated that for all age groups, the LH level in Group A were significantly higher than those in Group B. The endometrium may be exposed to high $E_{2}$ and LH levels, resulting in a decrease in endometrial receptivity [13]. 2) The earlier elevation in progesterone levels may have affected endometrial receptivity. 3) The GnRH-a induced LH surge lasted only $24 \mathrm{~h}$, which was significantly shorter than the LH surge in the natural cycle (48 h) [14].

More researches are needed to improve CPR of antagonist protocol. Some probable measures include: 1) A fixed drug-administration regimen should be employed. The advantages of it include the effective control of early LH surge, achievement of a sufficient oocytes, and enough high quality embryo [15]. 2) The luteal phase support protocol should be enhanced, especially when a GnRHa trigger is employed. [16].3) As stated in the guidelines of the European Society of Human Reproduction and Embryology, whole-embryo cryopreservation may be performed when a GnRH-a trigger is used in the antagonist protocol to prevent CPR decreases.

There were some limitations because it was a retrospective study. The small 
sample size of the study should be noted. Our results should be confirmed by further, adequately sized studies, or meta-analyzed along with similar studies.

\section{Conclusion}

This study has shown that the antagonist protocol could significant reduce pregnancy outcomes in young patients ( $<35$ years). Young patients with normal ovarian function can be treated with the agonist protocol, but the antagonist protocol should be considered in high ovarian responders (e.g., PCOS patients) to avoid OHSS. Older patients ( $>35$ years) could be treated with the antagonist protocol, which has fewer Gn administration days, lower Gn administration doses and lower total treatment costs, thus making it more acceptable to the patients.

\section{Competing Interests}

The authors declare that they have no competing interests.

\section{References}

[1] Porter, R.N., Smith, W., Craft, I.L., Abdulwahid, N.A. and Jacobs, H.S. (1984) Induction of Ovulation for In-Vitro Fertilisation Using Buserelin and Gonadotropins. Lancet, 2, 1284-1285. https://doi.org/10.1016/S0140-6736(84)92840-X

[2] Lainas, T.G., Sfontouris, I.A., Zorzovilis, I.Z., Petsas, G.K., Lainas, G.T., Alexopoulou, E., et al. (2010) Flexible GnRH Antagonist Protocol versus GnRH Agonist Long Protocol in Patients with Polycystic Ovary Syndrome Treated for IVF: A Prospective Randomised Controlled Trial (RCT). Human Reproduction, 25, 683-689. https://doi.org/10.1093/humrep/dep436

[3] Check, M.L., Check, J.H., Choel, J.K., Davies, E. and Kiefer, D. (2004) Effect of Antagonists vs Agonists on In Vitro Fertilization Outcome. Clinical and Experimental Obstetrics and Gynecology, 31, 257-259.

[4] Xing, W., Lin, H., Li, Y., Yang, D., Wang, W. and Zhang, Q. (2015) Is the GnRH Antagonist Protocol Effective at Preventing OHSS for Potentially High Responders Undergoing IVF/ICSI? PLoS One, 10, e0140286.

https://doi.org/10.1371/journal.pone.0140286

[5] Qiao, J., Lu, G., Zhang, H.W., Chen, H., Ma, C., Olofsson, J.I., et al. (2012) A Randomized Controlled Trial of the GnRH Antagonist Ganirelix in Chinese Normal Responders: High Efficacy and Pregnancy Rates. Gynecological Endocrinology, 28, 800-804. https://doi.org/10.3109/09513590.2012.665103

[6] Marci, R., Caserta, D., Lisi, F., Graziano, A., Soave, I., Lo Monte, G., et al. (2013) In Vitro Fertilization Stimulation Protocol for Normal Responder Patients. Gynecological Endocrinology, 29, 109-112. https://doi.org/10.3109/09513590.2012.712002

[7] Xiao, J., Chen, S., Zhang, C. and Chang, S. (2013) Effectiveness of GnRH Antagonist in the Treatment of Patients with Polycystic Ovary Syndrome Undergoing IVF: A Systematic Review and Meta Analysis. Gynecological Endocrinology, 29, 187-191. https://doi.org/10.3109/09513590.2012.736561

[8] Wang, H.Y., Li, Y.X., Dun, L.L., Xu, T.T., Hao, Y.J., Liu, H.Y., et al. (2013) Antinociceptive Effects of Matrine on Neuropathic Pain Induced by Chronic Constriction Injury. Pharmaceutical Biology, 51, 844-850. https://doi.org/10.3109/13880209.2013.767363 
[9] Pundir, J., Sunkara, S.K., El-Toukhy, T. and Khalaf, Y. (2012) Meta-Analysis of GnRH Antagonist Protocols: Do They Reduce the Risk of OHSS in PCOS? Reproductive BioMedicine Online, 24, 6-22. https://doi.org/10.1016/j.rbmo.2011.09.017

[10] Kolibianakis, E.M., Griesinger, G. and Venetis, C.A. (2012) GnRH Agonist for Triggering Final Oocyte Maturation: Time for a Critical Evaluation of Data. Human Reproduction Update, 18, 228-229; Author Reply 9-30. https://doi.org/10.1093/humupd/dmr055

[11] Al-Inany, H.G., Youssef, M.A., Aboulghar, M., Broekmans, F., Sterrenburg, M., Smit, J., et al. (2011) Gonadotrophin-Releasing Hormone Antagonists for Assisted Reproductive Technology. The Cochrane Database of Systematic Reviews, 2011, CD001750.

[12] Hershko Klement, A., Berkovitz, A., Wiser, A., Gonen, O., Amichay, K., Cohen, I., et al. (2015) GnRH-Antagonist Programming versus GnRH Agonist Protocol: A Randomized Trial. The European Journal of Obstetrics \& Gynecology and Reproductive Biology, 185, 170-173.

[13] Copperman, A.B. and Benadiva, C. (2013) Optimal Usage of the GnRH Antagonists: A Review of the Literature. Reproductive Biology and Endocrinology, 11, 20. https://doi.org/10.1186/1477-7827-11-20

[14] Vaisbuch, E., de Ziegler, D., Leong, M., Weissman, A. and Shoham, Z. (2014) Luteal-Phase Support in Assisted Reproduction Treatment: Real-Life Practices Reported Worldwide by an Updated Website-Based Survey. Reproductive BioMedicine Online, 28, 330-335.

[15] Oberye, J.J., Mannaerts, B.M., Huisman, J.A. and Timmer, C.J. (1999) Pharmacokinetic and Pharmacodynamic Characteristics of Ganirelix (Antagon/Orgalutran). Part II. Dose-Proportionality and Gonadotropin Suppression after Multiple Doses of Ganirelix in Healthy Female Volunteers. Fertility and Sterility, 72, 1006-1012.

[16] Youssef, M.A., Van der Veen, F., Al-Inany, H.G., Griesinger, G., Mochtar, M.H., Aboulfoutouh, I., et al. (2011) Gonadotropin-Releasing Hormone Agonist versus HCG for Oocyte Triggering in Antagonist Assisted Reproductive Technology Cycles. The Cochrane Database of Systematic Reviews, 2011, CD008046. https://doi.org/10.1002/14651858.CD008046.pub3 